Citation: Lambert R, Radford K, Smyth G, Morley M, Ahmed-Landeryou M (2014) Occupational therapy can flourish in the 21st century - a case for professional engagement with health economics. British Journal of Occupational Therapy, 77(5), 260-263. DOI:

\title{
Occupational therapy can flourish in the 21st century - a case for professional engagement with health economics
}

\author{
Rod Lambert ${ }^{1}$ Kate Radford ${ }^{2}$ Genevieve Smyth ${ }^{3}$, Mary Morley ${ }^{4}$ \& Musharrat Ahmed- \\ Landeryou $^{5}$ \\ ${ }^{1}$ Lecturer in Occupational Therapy and Senior Associate, Health Economics Consulting, University of \\ East Anglia, Faculty of Health, Norwich, Norfolk \\ ${ }^{2}$ Associate Professor in Rehabilitation, Research (Long-term conditions), University of Nottingham, \\ Division of Rehabilitation and Ageing, Nottingham \\ ${ }^{3}$ Professional Affairs Officer, College of Occupational Therapists, London \\ ${ }^{4}$ Director of Therapies, South West London and St George's Mental Health NHS Trust, London \\ ${ }^{5}$ Senior Lecturer, Occupational Therapy, London South Bank University, Allied Health Sciences, \\ London \\ Email: Dr Rod Lambert - lambert@uea.ac.uk

\section{Introduction}

In 1996, David Nelson delivered the Eleanor Clarke Slagle lecture, an honour bestowed by the American Occupational Therapy Association to a member who has creatively added to occupational therapy knowledge, boldly stating that:

The profession of occupational therapy will flourish [in the 21st Century] because occupation, its core, is so basic to human health yet so flexible, depending on the needs of the individual human being (Nelson 1997).

This positive and encouraging perspective was provided, however, before the collapse of the banking system and financial markets in 2008 (Mathieson and Stewart 2008). This financial collapse led to austerity measures in healthcare (Karanikolos et al 2013), which have influenced an already contentious debate about the financing and resourcing of healthcare (Appleby 2012, Appleby 2011). Demonstrating the clinical effectiveness of healthcare interventions is no longer sufficient for decision-makers to draw conclusions about how best to allocate scarce resources (Manns 2009). Nelson's perspective focused on how the profession defines and incor-porates occupation as the core of professional thinking. However, it is vital that occupational therapy demonstrates not only 
that it has evidence for ethical, coherent, and convincing professional theories/models, client satisfaction, and clinical evidence, but also provides robust evidence of cost-effective input to patient care and patient choice.

\section{College of Occupational Therapists 'summit' on health economics}

Following an agenda item on the College of Occupational Therapists (COT) English Board, the COT convened a summit, in May 2013, with a group of prominent therapists already engaged in the field to discuss how to improve the profession's understanding and use of health economics. Health economics is concerned with exploring efficiency, effectiveness, value and behaviour in health, and healthcare production and consump-tion (Drummond 2005, Fox-Rushby and Cairns 2005). For the profession this relates to evaluation of occupational therapy service/intervention:

A specific treatment is said to be 'cost-effective' if it gives greater health gain than could be achieved by using the resources in other ways (Harwood 2008).

This opinion piece is one of the outcomes of the summit. We conducted two brief reviews of the evidence, presented here for consideration and comment. The search was con-fined to PubMed; results are not presented as comprehensive but, rather, to demonstrate emerging patterns.

Literature reviews

Cost-effectiveness analyses consider one clinical outcome and the associated resource use and, therefore, also consider the costs to the service provider of providing the intervention (Drummond 2005). This represents the most frequently used form of economic evaluation in the health sector (Fox-Rushby and Cairns 2005). The first review investigated whether an increased emphasis on economic evaluation is observable in the literature during the period 1996-2012, identifying articles with 'cost-effectiveness' or 'cost effectiveness' in the title or abstract (Fig. 1).

Fig. 1. PubMed search for papers with 'cost-effectiveness' or 'cost effectiveness' in title or abstract, 1996-2012

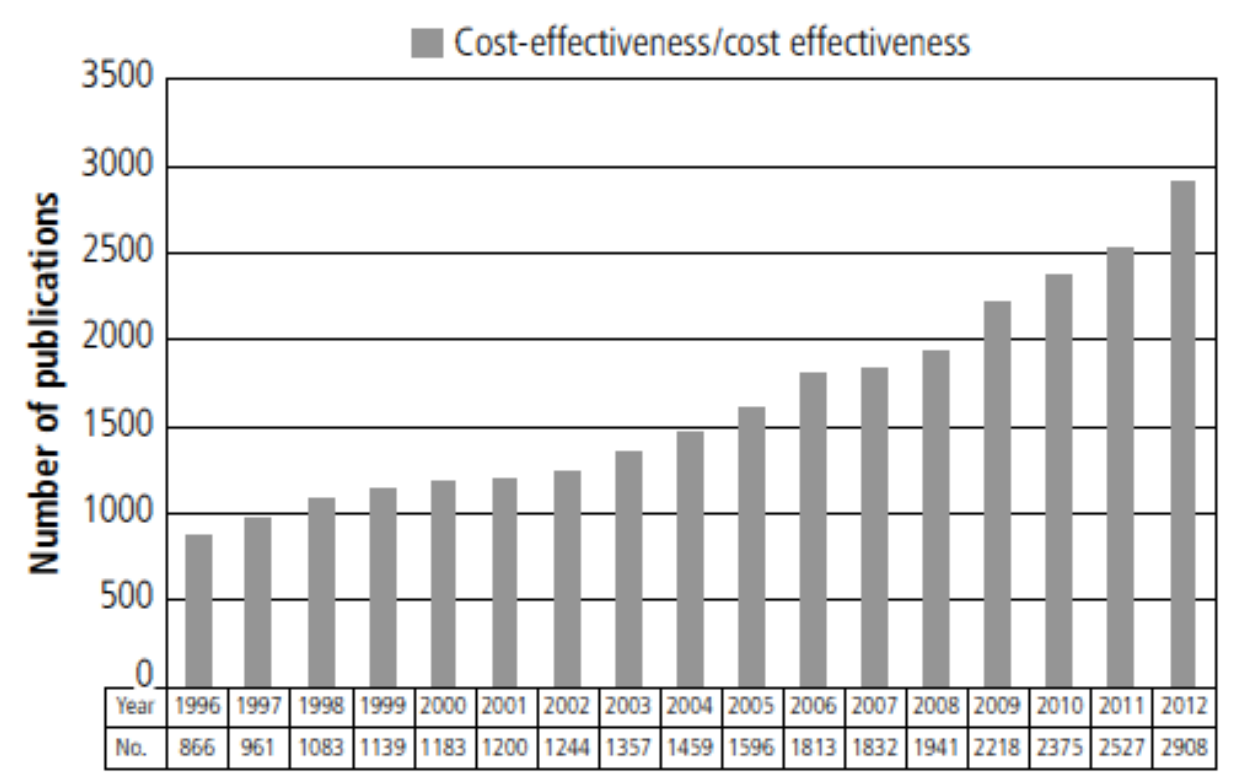


Note: data from PubMed.gov

As can be seen in Fig. 1, this search indicated an increase in published articles on cost-effectiveness of $236 \%$ (from 866 in 1996, to 2,908 articles in 2012). While there was a trend towards increasing numbers of articles on cost-effectiveness between 1996 and 2001 (39\% increase), and 2002 and 2007 (47\% increase), the four years between 2008 and 2012 saw an increase of 50\%, and still rising. This supports the need for evidence showing affordability in healthcare (Appleby 2012) and a growing need for cost-effectiveness analyses for health services and interventions.

Fig. 2. PubMed search for papers published 2012, with titles or abstracts suggesting economic evaluation, by profession

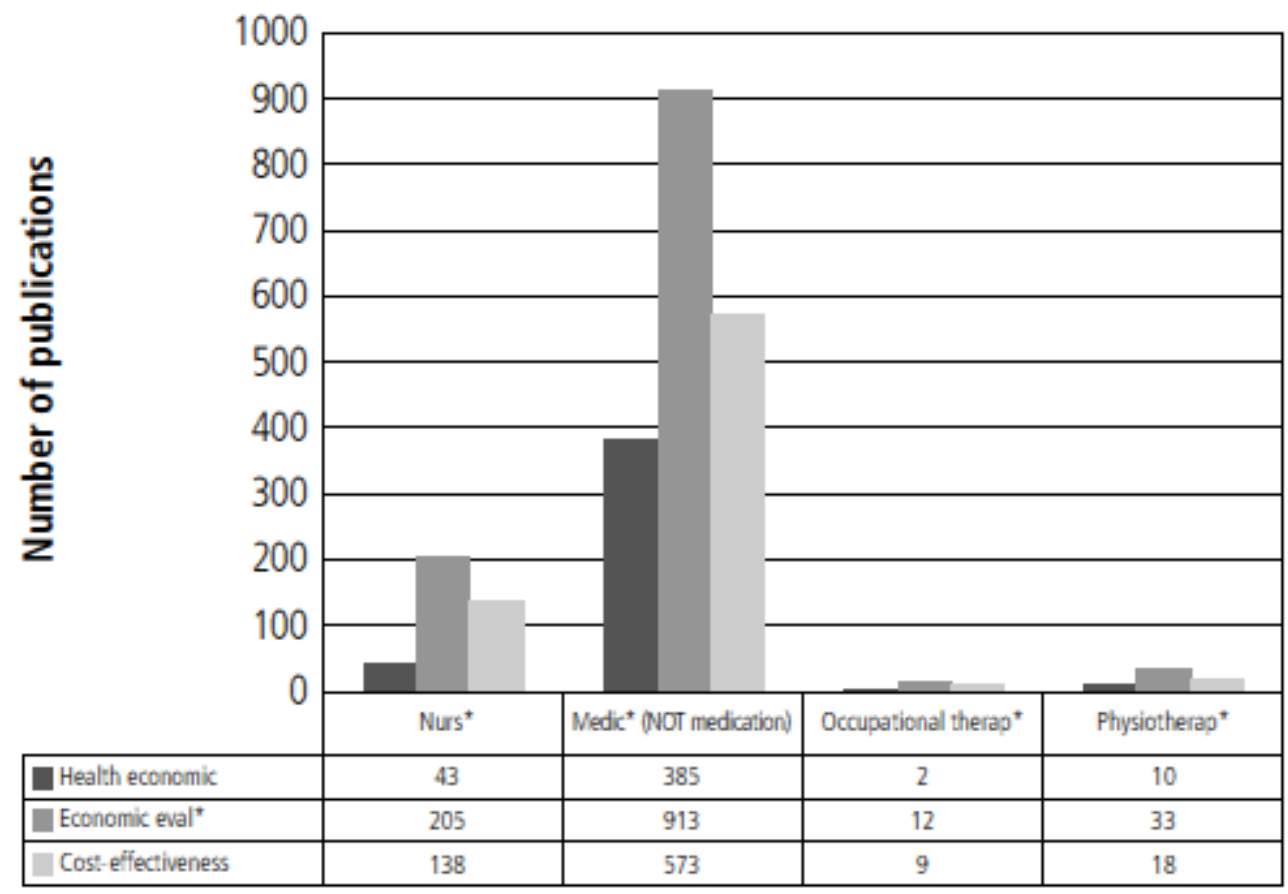

Note: data from PubMed.gov; *indicates wild-card character in search

If we accept this logic, then it follows that each of the healthcare professions needs to provide evidence that it is cost-effective. If occupational therapists are embracing this requirement, we should see an increased publication record of economic analyses, similar to that from the other profes-sions. To investigate this, we conducted a further search of PubMed using three main health economic terms in the title/abstract of articles.

\section{Filtering the search for professions}

Fig. 2 shows that from a total search resulting in 1,671 articles with 'health economic' in the title or abstract, 385 included 'medic* (NOT medication)' (the * wildcard ensuring that the filter captured 'medical' input and 'medical' conditions and excluding a 'medication' focus), 43 included 'nurs*' (to capture 'nurses' and 'nursing'), but only 2 included 'occupational therap*' (to capture both 'therapy' 
and 'therapist') and a further 10 'physiotherap*' (to capture 'physiotherapy' and 'physiotherapist'). This pattern was consistently applied for 'economic eval*' and either 'cost-effectiveness' or 'cost effectiveness' (for the year 2012 only; the date restriction was deemed necessary for this category, due to the high number of 'hits' achieved under 'medic* (NOT medication)', $n=5,959$ when unrestricted by date. In contrast, when unrestricted by date in this last category, there were 1,495 'hits' for 'nurs*', 55 for 'occupational therap*' and 149 for 'physiotherap*'). An important difference is that, while physiotherapy also lags significantly behind medicine and nursing, it has still pub-lished between twice and three times the number of economic evaluations in PubMed than occupational therapy.

\section{The need for more occupational therapy-based research}

This 'review' does not to pretend to be rigorous, and was simply a means of gaining a general overview of the relative difference in the amount of health economic literature between professions, and what appears to be a lack of literature on this topic specifically issuing from occupational therapy. Our profession has a long tradition of producing good qualitative research and evaluation, but the sheer volume of this leaves us at risk of perceiving ourselves and being perceived externally as a monoculture. It is recognized that qualitative evidence can be used productively within health economic decision making (Coast 2009, Coast et al 2004, Coast 1999). However, the key to survival is diversity and adaptation to new health and social care environments. This informal review suggests that occupational therapy is lagging behind in the publication of good quality scientific research that lends itself to economic evaluation. If we continue in this manner, other professions, other services, and other interventions will demonstrate to decision-makers and commissioners that they are costeffective. If we remain unable to demonstrate the same for occupational therapy we will founder, and the profession itself will become at risk for its survival.

\section{Moving forward}

In the short term, we need to increase the awareness and use of health economics in everyday practice and in service evalua-tions. This is likely to require ways of improving knowledge, understanding, and skills in the principles behind health eco-nomics. These include accessing available expertise in health economics through private consultancies or academic research units (usually based in universities) that may help to provide the support needed both in planning and implementing economic evaluation. Short courses, MSc modules, and programmes of study are available, and advice and support from health economists and health economics consultancies may offer a short-term route to incorporating health economics into research and service evaluation within a reasonable timescale.

In the medium term, investing in continuing professional development (CPD) for existing staff on health economics through well recognized MSc programmes would raise the confidence level of staff to undertake research and service evaluations from a health economics perspective.

In the longer term, it is necessary to incorporate some of these health economic principles into both the research and professional development elements of occupational therapy training programmes at undergraduate and pre-registration MSc levels. 
An overarching aim of these strategies should be to increase the published evidence base for the cost-effectiveness of occu-pational therapy interventions. Now that BJOT has achieved its first Impact Factor (1.096), a previous barrier to publication of good quality research in BJOT has now been removed.

\section{Summary}

The purpose of this opinion piece is to raise awareness of the issues, rather than to provide insight into the methods needed to engage in health economic analyses. However, if we are to be able to not only evaluate the clinical benefits of interventions but also consider the economic consequences, we need to understand the costs involved in delivering our services. Although local differences will always need to be considered, sources such as the annual update on unit costs provided by the Personal Social Services Research Unit at the University of Kent (Curtis 2012) often provide a good initial indicator.

To realise Nelson's prediction of a sustainable future for the profession, occupational therapists need to understand their position as part of a wider scientific health and social care community. The climate of increasing scrutiny requires services and interventions that show not only clinical efficacy and sound reasoning, but also demonstrate efficient use of resources. In response to this climate, recent publications have emerged examining the cost effectiveness of occupa-tional therapy interventions (Clark et al 2012, Irvine et al 2010; Lambert et al 2010, Sackley et al 2012), though improved quality and quantity of publications are needed to underpin the profession's work.

To flourish in a world that increasingly focuses on cost-effectiveness, economic evaluation provides a range of approaches, tools, and analytical frameworks that offer a way forward.

\section{References}

Appleby J (2012) Rises in healthcare spending: where will it end? British Medical Journal, 345, e7127.

Appleby J (2011) Can we afford the NHS in future? British Medical Journal, 343, d4321.

Clark F, Jackson J, Carlson M, Chou CP, Cherry BJ, Jordan-Marsh M, ... Azen SP (2012) Effectiveness of a lifestyle intervention in promoting the well-being of independently living older people: results of the Well Elderly 2 Randomised Controlled Trial. Journal of Epidemiology and Community Health, 66(11), 782-790.

Coast J (2009) Maximisation in extra-welfarism: a critique of the current position in health economics. Social Science \& Medicine, 69(5), 786-792.

Coast J (1999) The appropriate uses of qualitative methods in health economics. Health Economics, 8(4), 345-353.

Coast J, McDonald R, Baker R (2004) Issues arising from the use of qualitative methods in health economics. Journal of Health Services Research \& Policy, 9(3), 171-176.

Curtis L (2012) Unit Costs of Health and Social Care 2012. Canterbury, Kent: Personal Social Services Research Unit, University of Kent. 
Drummond MF, Sculpher MJ, Torrance GW, O'Brien BJ, Stoddart GL (2005) Methods for the economic evaluation of health care programmes. 3rd ed. Oxford: Oxford University Press.

Fox-Rushby JA, Cairns J (2005) Economic evaluation: understanding public health. Maidenhead: Open University Press.

Harwood RH (2008) Economic evaluations of complex services for older people. Age and Ageing, 37(5), 491-493.

Irvine L, Conroy SP, Sach T, Gladman JR, Harwood RH, Kendrick D, ... Masud T (2010) Cost-

effectiveness of a day hospital falls prevention programme for screened community-dwelling older people at high risk of falls. Age and Ageing, 39(6), 710-716.

Karanikolos M, Mladovsky P, Cylus J, Thomson S, Basu S, Stuckler D, Mackenbach JP, McKee M (2013) Financial crisis, austerity, and health in Europe. Lancet, 381(9874), 1323-1331.

Lambert RA, Lorgelly P, Harvey I, Poland F (2010) Cost-effectiveness analysis of an occupational therapy-led lifestyle approach and routine general practitioner's care for panic disorder. Social Psychiatry and Psychiatric Epidemiology, 45(7), 741-750.

Manns BJ (2009) The role of health economics within clinical research. Methods in Molecular Biology, $473,235-250$.

Mathieson N, Stewart H (2008) Three weeks that changed the world. The Observer, 28-12-2008.

Nelson DL (1997) Why the profession of occupational therapy will flourish in the 21st century. The 1996 Eleanor Clarke Slagle Lecture. American Journal of Occupational Therapy, 51(1), 11-24.

Sackley CM, Burton CR, Herron-Marx S, Lett K, Mant J, Roalfe AK, ... Feltham MG (2012) A cluster randomised controlled trial of an occupational therapy intervention for residents with stroke living in UK care homes (OTCH): study protocol. BMC Neurology, 12(1), 52 